\title{
Optimization of Organ Conductivity for the Forward Problem of Electrocardiography
}

\author{
Laura Bear ${ }^{1,2,3}$, Rémi Dubois ${ }^{1,2,3}$, Nejib Zemzemi ${ }^{1,4}$ \\ ${ }^{1}$ IHU Liryc, Electrophysiology and Heart Modeling Institute, fondation Bordeaux Université, \\ Pessac- Bordeaux, France \\ ${ }^{2}$ Univ. Bordeaux, Centre de recherche Cardio-Thoracique de Bordeaux, U1045, Bordeaux, France \\ ${ }^{3}$ INSERM, Centre de recherche Cardio-Thoracique de Bordeaux, U1045, Bordeaux, France \\ ${ }^{4}$ CARMEN Research Team, INRIA, Talence, France
}

\begin{abstract}
This study presents an empirical approach to optimize conductivities within a torso model given simultaneous epicardial and body surface potential recordings. The conductivities of the lungs, skeletal muscle and torso cavity were estimated within a forward model by minimizing the relative error between computed and reference torso potentials using a standard gradientbased approach. The sensitivity of this approach was evaluated over different levels of geometric error and signal noise, and the gradient of the cost function was determined using both 1) finite differences and 2) an adjoint method.

All conductivities were accurately estimated $(<10 \%$ difference in value) with up to $0.20 \mathrm{mV}$ signal noise and all levels of electrode localization error (up to $2.56 \mathrm{~cm}$ ) using a finite difference approach. While the adjoint approach was more computationally efficient, a finite difference approach was more stable across different signals and more robust to noise.
\end{abstract}

\section{Introduction}

The forward problem of electrocardiography defines the relationship between epicardial and body surface potentials. This relationship is fundamental to the inverse problem (non-invasive imaging of cardiac electrical activity from body surface measurements). As the inverse problem is ill-posed, it is important that the forward model is identified accurately.

In previous studies comparing directly recorded and forward computed body surface potentials in-vivo, it was shown that forward models incorporating inhomogeneous structures were more accurate than homogeneous models [1,2], improving both the magnitude and pattern of body surface potentials. Despite this, there still remained a difference between the forward and recorded body surface potentials. Although including further structural details into these forward models may improve them, such as by incorporating muscle fibre directions, it is often computationally and technically infeasible to do so.

The conductivity values assigned to different organs in most forward models are based on three studies [3-5], with the values varying substantially between them. Previously, it has been shown that error in the conductivity values assigned can significantly affect body surface potentials, and in different ways depending on the organ [6].

Theoretically, the optimal conductivity for each region in a forward model can be found given a data set with simultaneous measures of epicardial and body surface potentials. That is, by defining one organ as having a constant conductivity, its ratio to other organ conductivities can be optimized to best match predicted and observed torso potentials. The basis of this approach is empirical rather than physiological. Nonetheless, identification of an optimized transfer matrix would provide a better forward model than both a uniform isotropic model and a more complex physiologically based one. This study examines such a method for optimizing conductivities using previously obtained invivo experimental data [1].

\section{Methods}

\subsection{Experimental data}

In-vivo, experimental data came from an anesthetized, closed-chest pig [1]. Epicardial and torso potentials were recorded simultaneously using an elastic "sock" (239 unipolar electrodes) and flexible strips attached to the body surface (184 electrodes). Upon completion, the heart was arrested and MR images acquired. The heart was excised and perfusion-fixed. Epicardial electrode locations were captured with a multi-axis digitizing arm. MRI contrast markers placed on the "sock" and body surface strips were localized in the MR images and used to register electrode locations. 


\subsection{Geometric and computational model}

In order to define a test data set, a volume conductor model was developed using post-mortem MRI. An anatomically realistic generic model of a pig was customized to data clouds of the epicardial, lungs, fat and skeletal muscle surfaces, using a non-linear fitting procedure [7]. Linear-triangular surface meshes were then coupled together to create a finite element volume conductor model (Fig 1).

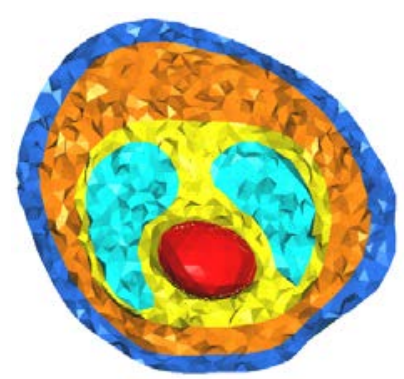

Fig 1. Cranial view of the torso model. Regions include the epicardium (red) lungs (light blue), cavity (yellow), skeletal muscle (orange) and subcutaneous fat (dark blue).

The torso domain is denoted by $\Omega$, covering the volume between the epicardium and body surface.

$$
\Omega=\cup_{i \in\{f, m, l, c\}} \Omega_{i},
$$

where $\Omega_{f}, \Omega_{m}, \Omega_{l}, \Omega_{c}$ are the fat, skeletal muscle, lung and cavity volumes respectively. The conductivity in each region was defined as $\sigma_{f}=0.04, \sigma_{m}=0.40, \sigma_{l}=0.05, \sigma_{c}=$ $0.22 \mathrm{mSmm}^{-1}$, consistent with the literature [3-5]. The boundary of the torso domain is defined by $\partial \Omega=\Sigma U$ $\Gamma_{\text {ext }}$, where $\Sigma$ represents the epicardial surface and $\Gamma_{\text {ext }}$ the external boundary of the body surface. We denote the torso potential in $\Omega$ by $u_{T}$. By solving Laplace's equation over this volume using the finite element method, we define the linear relationship: $d=A h$. At each time step, reference potentials, $d$, at 180 electrode locations on $\Gamma_{\text {ext }}$ were computed from the measured potentials, h on $\Sigma$.

\subsection{Optimization procedure}

The test data provides the potential values on both $\Sigma$ and $\Gamma_{\text {ext }}$. Moreover, we know that the current flux over the body surface is equal to zero. In order to estimate the values of each organ conductivity, we construct the following quantity of interest.

$$
\left\{\begin{array}{c}
\mathrm{I}\left(\sigma_{f}, \sigma_{m}, \sigma_{l}, \sigma_{c}\right)=\frac{1}{2}\left\|u_{T}-d\right\|_{L^{2}\left(\Gamma_{e x t}\right)}^{2}, \\
\text { with } u_{T} \text { solution of: } \\
\left\{\begin{array}{c}
\operatorname{div}\left(\sigma_{T} \nabla u_{T}\right)=0, \text { in } \Omega, \\
u_{T}=h, \text { on } \Sigma, \\
\sigma \nabla u_{T} \cdot \boldsymbol{n}_{T}=0, \text { on } \Gamma_{\text {ext }}
\end{array}\right.
\end{array}\right.
$$

By minimizing the cost function, $\mathrm{I}\left(\sigma_{f}, \sigma_{m}, \sigma_{l}, \sigma_{c}\right)$, we find the optimal values of the conductivity for all four organs. Here, the fmincon function of Matlab 2013b was used. By default, this function approximates the derivative of I over $\sigma_{i}$ for $i \in\{f, m, l, c\}$ using a finite difference approach $\left(\mathrm{Grad}_{\mathrm{FD}}\right)$.

In the second half of this paper, we compare $\mathrm{Grad}_{\mathrm{FD}}$ to an alternative method, Grad $_{\text {adj, }}$, where the derivative of I over $\sigma_{i}$ is computed directly giving

$$
\frac{\partial I}{\partial \sigma_{i}}\left(\sigma_{f}, \sigma_{m}, \sigma_{l}, \sigma_{c}\right)=\int_{\Gamma_{e x t}} \frac{\partial \mathrm{u}_{T}}{\partial \sigma_{i}}\left(\mathrm{u}_{T}-d\right)
$$

As the derivative of $\mathrm{u}_{T}$ cannot be computed directly, an adjoint method was used. That is, $H^{1}(\Omega)$ is denoted by the set of functions $\emptyset: \Omega \rightarrow \mathbb{R}$, such that $\int_{\Omega} \emptyset^{2}<\infty$ and $\int_{\Omega}|\nabla \emptyset|^{2}<\infty . H_{\Sigma}^{1}(\Omega)$ is also denoted by the set of functions $\psi \in H^{1}(\Omega)$ such that $\psi / \Sigma=0$. For every $\left(\sigma_{f}, \sigma_{m}, \sigma_{l}, \sigma_{c}, u, \lambda\right) \in\left(\mathbb{R}^{+}\right)^{4} H^{1}(\Omega) \times H_{\Sigma}^{1}(\Omega)$, the Lagrangian function is defined as follows

$$
L\left(\sigma_{f}, \sigma_{m}, \sigma_{l}, \sigma_{c}, u, \lambda\right)=\frac{1}{2}\left\|u_{T}-d\right\|_{L^{2}\left(\Gamma_{e x t}\right)}^{2}+\int_{\Omega} \sigma_{T} \nabla u \nabla \lambda
$$

For every $\lambda \in H_{\Sigma}^{1}(\Omega)$ this gives

$$
\mathrm{I}\left(\sigma_{f}, \sigma_{m}, \sigma_{l}, \sigma_{c}\right)=L\left(\sigma_{f}, \sigma_{m}, \sigma_{l}, \sigma_{c}, u, \lambda\right)
$$

The gradient of I with respect to $\sigma_{i}$ is then given by

$$
\left\{\begin{array}{c}
\frac{\partial \mathrm{I}\left(\sigma_{f}, \sigma_{m}, \sigma_{l}, \sigma_{c}\right)}{\partial \sigma_{i}}=\int_{\Omega_{i}} \nabla u_{T} \nabla \lambda, \\
\text { with } \lambda \text { solution of }: \\
\operatorname{div}\left(\sigma_{T} \nabla \lambda\right)=0, \text { in } \Omega, \\
\lambda=0, \text { on } \Sigma, \\
\sigma \nabla \lambda \cdot \boldsymbol{n}_{T}=-\left(u_{T}-d\right), \text { on } \Gamma_{\text {ext }}
\end{array}\right.
$$

This method allows us to obtain the derivative of the objective function over the four conductivity parameters, by only solving two Laplace equations: The first is the state equation to obtain $\mathbf{u}_{T}$ and the second is the adjoint equation to obtain $\lambda$. The derivative is then obtained by integrating the scalar product of the gradients of $\mathbf{u}_{T}$ and $\lambda$ over each of the four organs domains.

\subsection{Method of analysis}

The sensitivity of the optimization procedure was tested by varying levels of signal noise (SN) on d, and torso electrode localization error (LE). SN was created using a random number generator with a standard deviation (SD) from 0.002 to $0.512 \mathrm{mV}$. The direction of LE for each electrode was defined by picking a random point on the surface of a unit sphere, with the distance defined with a mean from 0.02 to $2.56 \mathrm{~cm}$ across all electrodes. Conductivities were optimized for each level of error, and for six different time points spanning ventricular depolarization. In addition, conductivities were optimized combining a $\mathrm{SN}$ of $0.05 \mathrm{mV}$ and a LE 9 $\mathrm{mm}$. These values were estimated to be the level of error in the original data set.

The initial conductivities used to start optimization 
were defined using a Monte Carlo simulation. That is, simulations were run using four values of each conductivity ( $\sigma_{\mathrm{f}}$ kept constant at $0.04 \mathrm{mSmm}^{-1}$ ) ranging from $\pm 50 \%$ of the max/min values as compiled from the literature. The conductivities resulting in the lowest cost function were used as the initial conditions.

The accuracy of optimized conductivities was evaluated using the relative error (RE). In addition, the speed of computation was evaluated using the number of iterations for computation. To determine differences, a paired t-test was used for normally distributed data, and a two-sided Wilcoxon signed rank test for non-normal. Statistical significance was accepted for $\mathrm{p}<0.05$.

\section{Results}

\subsection{Sensitivity analysis}

Given the correct fat conductivity, the Monte Carlo simulations yielded initial conductivities of $\sigma_{\mathrm{l}}=0.06, \sigma_{\mathrm{m}}=$ $0.35, \sigma_{\mathrm{c}}=0.13 \mathrm{mSmm}^{-1}$, for all levels of signals noise, vest error, and for all time steps. In the following analysis, Grad $\mathrm{FD}$ was used to define the gradient during optimization.

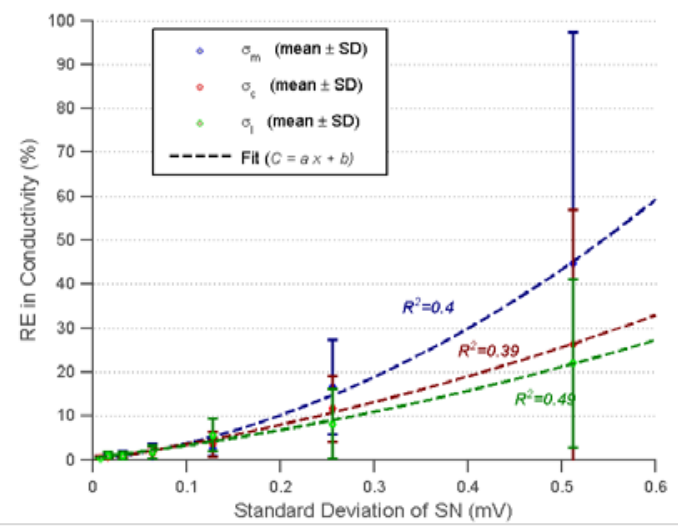

(a)

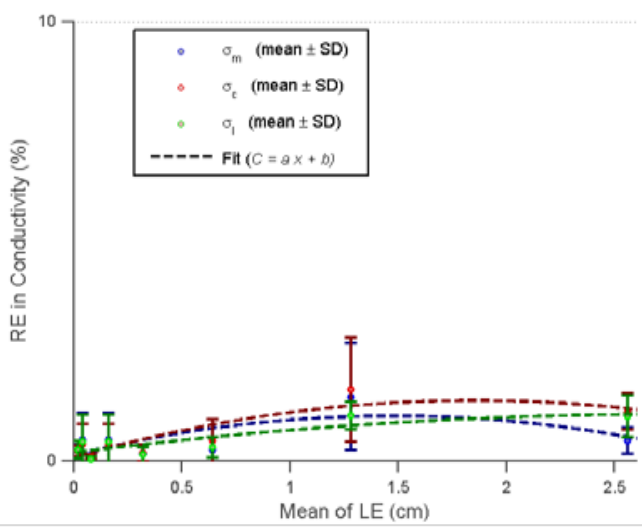

(b)

Fig 2. RE in conductivity over (a) SN and (b) LE.

As expected, the accuracy of optimization decreased with increasing levels of SN and LE (Fig. 2). All three conductivities were accurately estimated ( $\mathrm{RE}<10 \%)$ for up to $0.20 \mathrm{mV} \mathrm{SN}$ and for all levels of LE. The accuracy was also dependent on the signal used as demonstrated by the large SD of the results for each error level.

Table 1 Optimized conductivity values and RE when combining SN of $0.05 \mathrm{mV}$ and LE of $0.9 \mathrm{~cm}$, presented as median [inter quartile range].

\begin{tabular}{cccc}
\hline & $\boldsymbol{\sigma}_{\mathbf{m}}$ & $\boldsymbol{\sigma}_{\mathbf{l}}$ & $\boldsymbol{\sigma}_{\mathbf{c}}$ \\
\hline RE (\%) & $1.4[2.8]$ & $1.7[1.9]$ & $1.0[1.9]$ \\
\hline
\end{tabular}

Conductivities optimized combining SN (0.05 mV) and LE (9 mm) were accurately estimated for all signals used (Table 1).

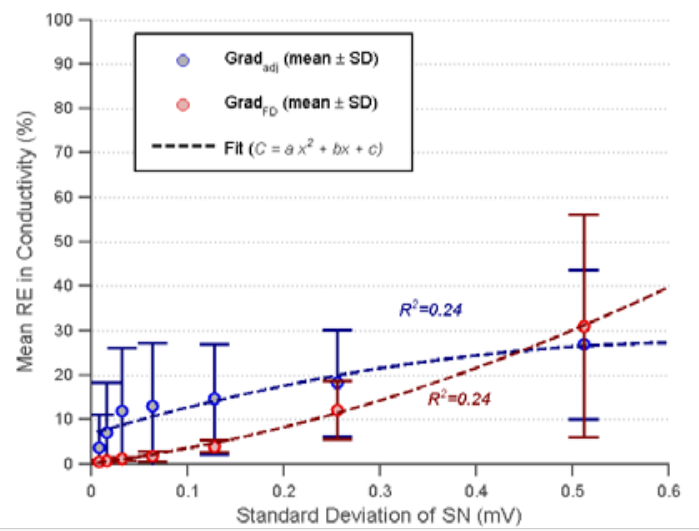

(a)

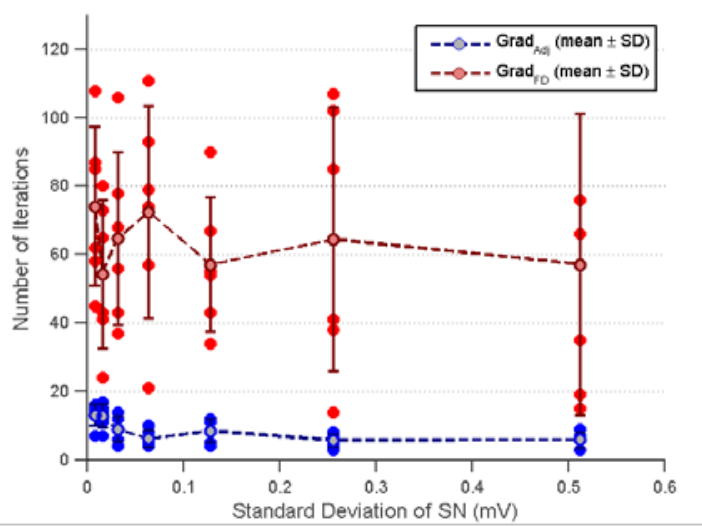

(b)

Fig 3. Comparison of Grad $\mathrm{FD}$ and Grad $_{\text {adj }}$ over SN using (a) mean RE in conductivity and (b) number of iterations for computation.

\subsection{Adjoint method for gradient}

The accuracy and speed of optimization using an adjoint apparoach to compute the gradient $\left(\mathrm{Grad}_{\mathrm{adj}}\right)$ were compared to $\mathrm{Grad}_{\mathrm{FD}}$. Given results are more sensitive to it, this comparison was performed over different levels of $\mathrm{SN}$. Fig. 3a presents the mean RE in conductivity against 
increasing SN for both methods. For all error levels except the greatest, the conductivities were more accurately estimated, and the SD smaller using Grad $\mathrm{FD}$. Overall, Grad $_{a d j}$ resulted in a significantly larger average error in conductivity $(\mathrm{p}<0.0043)$ than Grad $_{\mathrm{FD}}$.

Fig $3 \mathrm{~b}$ shows the number of iterations necessary to converge to a solution for each method. From this, we can see Grad $_{\mathrm{adj}}$ is substantially more computationally efficient than $\operatorname{Grad}_{\mathrm{FD}}$, requiring 45 to 64 fewer iterations to converge to a solution for all levels of signal noise. Interestingly, the number of iterations remains relatively stable with increasing noise levels for both methods.

\section{Discussion}

In this study, we have presented a method for optimizing the conductivity of different organs in a torso model, given simultaneous recordings of epicardial and torso potentials. Here, conductivities of 3 organs were successfully computed under typical signal noise and geometric error levels. A sensitivity analysis revealed the conductivities could be computed to within $10 \%$ of their true values when the standard deviation of signal noise was less than $0.20 \mathrm{mV}$ and the mean electrode localization error less than $2.56 \mathrm{~cm}$.

The accuracy of the final conductivity values was dependent on the signal selected for optimization, demonstrated by an increase in SD with error. Further analysis showed that signals taken near the start and end of depolarization were typically less accurate than those near the middle of depolarization. Thus, care must be taken when selecting the signals used for optimization as the method is dependent not only on the noise level but the signal-to-noise ratio itself.

Generally, optimization is more robust, and faster when you include directly computed gradients. Here, while Grad $_{\text {adj }}$ was more computationally efficient than the standard Grad ${ }_{\mathrm{FD}}$ approach provided by Matlab, it was less robust to signal noise. Despite having not converged to the exact conductivity using Grad $_{\mathrm{adj}}$, the final cost function values were not largely different from those using Grad ${ }_{\mathrm{FD}}$, where conductivities accurately converged. Thus, we could assume the gradient calculated using $\mathrm{Grad}_{\mathrm{adj}}$ typically resulted in small step sizes, and the optimization process was exited due to a step size or a change in cost function below threshold. Changes to these thresholds would increase the computation time, with no guarantee of a substantially improved convergence.

Previous studies have shown structure-based inhomogeneous models have proven to be less robust than homogeneous models in inverse simulations [8]. That is, although more accurate physiologically, inhomogeneous models are also more prone to error. However, here we have shown that an optimized transfer matrix can be identified, providing a better forward model than a uniform isotropic volume conductor, and potentially more robust inverse solutions than complex physiologically based inhomogeneous models. Thus these methods could be of clinically to improve current electrocardiographic techniques.

Whilst a full set of epicardial potentials is typically unavailable clinically, an electrical current could be applied to the heart in a known location during isoelectric periods. Given body surface potentials recorded concurrently and patient-specific MRI, the organ conductivities within a patient could theoretically be estimated. Further analysis in this direction is currently underway using the experimental data outlined.

\section{Acknowledgements}

This work was supported in part by the French National Research Agency under Contract ANR-10IAHU04-LIRYC and by the CORDIS3D IRSES-GA2013-317767 grant. Laura Bear was supported by grant ANR-10-IAHU-04.

\section{References}

[1] Bear LR, Cheng LK, LeGrice IJ, Sands GB, Lever NA, Paterson DJ, et al. Forward Problem of Electrocardiography. Circ Arrhythmia Electrophysiol. 2015;8(3):677-84.

[2] Stanley PC, Pilkington TC, Morrow MN, Ideker RE. An assessment of variable thickness and fiber orientation of the skeletal muscle layer on electrocardiographic calculations. IEEE Trans Biomed Eng. 1991 Nov;38(11):1069-76.

[3] Geddes LA, Baker LE. The specific resistance of biological material - a compendium of data for the biomedical engineer and physiologist. Med Biol Eng. 1967;5:271-93.

[4] Rush S, Abildskov JA, McFee R. Resistivity of body tissues at low frequencies. Circ Res. 1963;12:40-50.

[5] Foster KR, Schwan HP. Dielectric properties of tissue and biological materials: A critical review. Crit Rev Biomed Eng. 1989;17(1):25-104.

[6] Geneser SE, Kirby RM, Macleod R. Application of stochastic finite element methods to study the sensitivity of ECG forward modeling to organ conductivity. IEEE Trans Biomed Eng. 2008;55(1):31-40.

[7] Bradley CP, Pullan AJ, Hunter PJ. Geometric modeling of the human torso using cubic hermite elements. Ann Biomed Eng. 1997;25(1):96-111.

[8] Ramanathan C, Rudy Y. Electrocardiographic imaging: II. Effect of torso inhomogeneities on noninvasive reconstruction of epicardial potentials, electrograms, and isochrones. J Card Electrophysiol. 2001;12(2):241-52.

Address for correspondence:

Laura Bear.

IHU-LIRYC, Avenue du Haut Lévèque, 33600 Pessac, France. laura.bear@ihu-liryc.fr 